\title{
Structural Relaxation in Nanometer Thin Layers of Glycerol
}

\author{
S. Capponi, ${ }^{\dagger}$ S. Napolitano ${ }^{\dagger}$ N. R. Behrnd, ${ }^{\ddagger}$ G. Couderc,,${ }^{\ddagger}$ J. Hulliger,${ }^{\ddagger}$ and M. Wübbenhorst $*, \dagger$ \\ Department of Physics and Astronomy, Katholieke Universiteit Leuven, Celestijnenlaan 200D, Leuven, 3001, Belgium, and \\ Department of Chemistry and Biochemistry, University of Berne, Freiestr. 3, 3012 Berne, Switzerland
}

Received: August 27, 2010

\begin{abstract}
The molecular dynamics in nanometer thin films of glycerol was investigated upon thickness reduction by combining organic molecular deposition with in situ broadband dielectric spectroscopy. Changes in the cooperative dynamics with respect to bulk glycerol were observed for films of thicknesses down to $1.6 \mathrm{~nm}$ (corresponding to roughly three molecular layers). Systematic investigation revealed no pure size effects addressable merely to geometrical constraints. However, an increase in the glass transition temperature by $3.5 \mathrm{~K}$ was observed for the thinnest film, indicating the presence of a layer with reduced mobility in close proximity to the substrate. The impact of both the upper and lower interfaces has been disentangled by measurements performed during slow desorption. Moreover, proof is given for the existence of a layer with enhanced mobility in the vicinity of the free surface enslaved to the dynamics of the rest of the film.
\end{abstract}

The cooperative dynamics in nanoconfined glass formers is a topic of high interest in condensed matter physics for more than two decades. One of the most controversial aspects concerns the existence and the spatial extent of a critical characteristic dimension, below which the geometrical restriction would cause a transition from a bulk like cooperative behavior to faster dynamics characterized by a temperature independent activation barrier (finite size effects). ${ }^{1-4}$ Despite striking differences in the experimental conditions, a common finding emerging from these studies is that the overall confinement effects on the structural dynamics are also influenced by the presence of attractive or repulsive interactions ${ }^{2,5-7}$ between the glass-forming liquid and the solid host (interfacial effects).$^{8-10}$ In order to achieve a reliable and complete picture, both contributions need to be disentangled accurately. In this respect ultrathin films represent probably the most straightforward geometry, providing the possibility to control independently the confinement dimension, the film thickness, and the surface interactions.

In this paper we study the evolution of the cooperative dynamics upon thickness reduction in ultrathin films of glycerol investigated by broadband dielectric spectroscopy performed in situ and in real-time. The experimental approach we adopted takes advantage of organic molecular beam deposition (OMBD) to grow films on interdigitated comb electrodes (IDE), ${ }^{11-13}$ permitting a continuous measurement of the dielectric response of the sample. On the basis of this methodology, we have monitored the structural relaxation of glycerol films of different thicknesses, down to $0.7 \mathrm{~nm},{ }^{14}$ as a function of the temperature. In addition, isothermal experiments were performed that gave accurate access to the evolution of the distribution of relaxation times during continuous thinning of the film. The latter approach resulted, particularly powerfully, in the search for a characteristic film thickness, at which deviations in the relaxation parameters would indicate the existence of a possible intrinsic length scale in the liquid. Interfacial effects have also been investigated. In particular, the impact of the free surface on the effective

\footnotetext{
* Corresponding author. E-mail: wubbenhorst@fys.kuleuven.be.

${ }^{\dagger}$ Katholieke Universiteit Leuven.

* University of Berne.
}

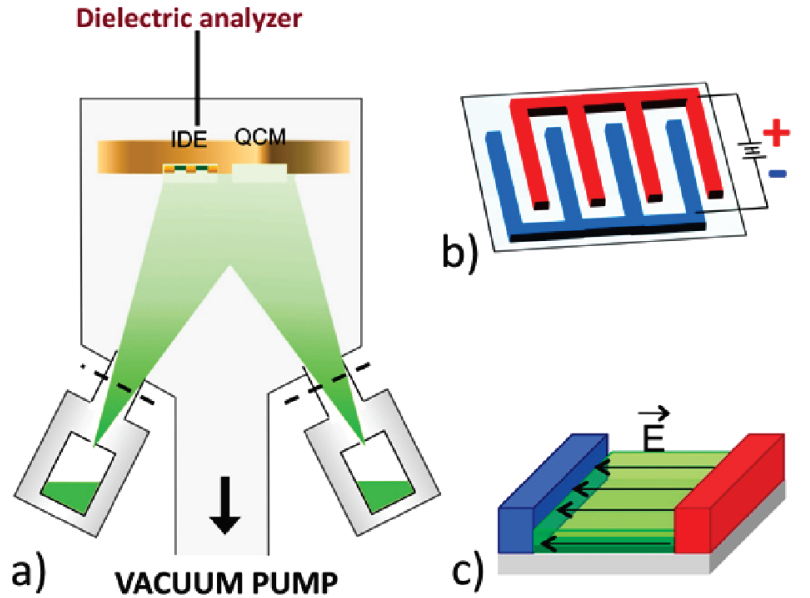

Figure 1. Sketch of the experimental set up (a). The structure of the IDE is represented for an empty sensor (b) and in the presence of a film (c).

dynamics of the system has been quantified by comparison with a capped film where this contribution is suppressed. ${ }^{15}$

Though no finite size effects (transition from cooperative to single molecule dynamics) have been observed in any of the measured samples, for films below $3 \mathrm{~nm}$, we found a broadening in the loss peak accompanied by a slowdown in the dynamics for samples of a thickness corresponding to $\sim 3$ molecular layers $(1.6 \mathrm{~nm})$. Both effects have been ascribed to the presence of attractive interactions between glycerol and the quartz substrate of the IDE sensor.

Figure 1 shows a schematic representation of the experimental set up. The material used for deposition is stored in a crucible provided with a very narrow hole (Knudsen cell) that is attached to an UHV vacuum chamber, maintaining a pressure in the order of $p \sim 10^{-8}$ mbar. The flux of the molecular beam hitting the IDE sensor is adjusted by varying the temperature of the Knudsen cell and thus the vapor pressure of the source. Due to its open electrode configuration, the sensor is constantly accessible for the incoming molecules, forming a liquid film between the fingers stabilized via strong $\mathrm{H}$-bonds to the oxide substrate of the IDE. Simultaneously, the thickness of the 


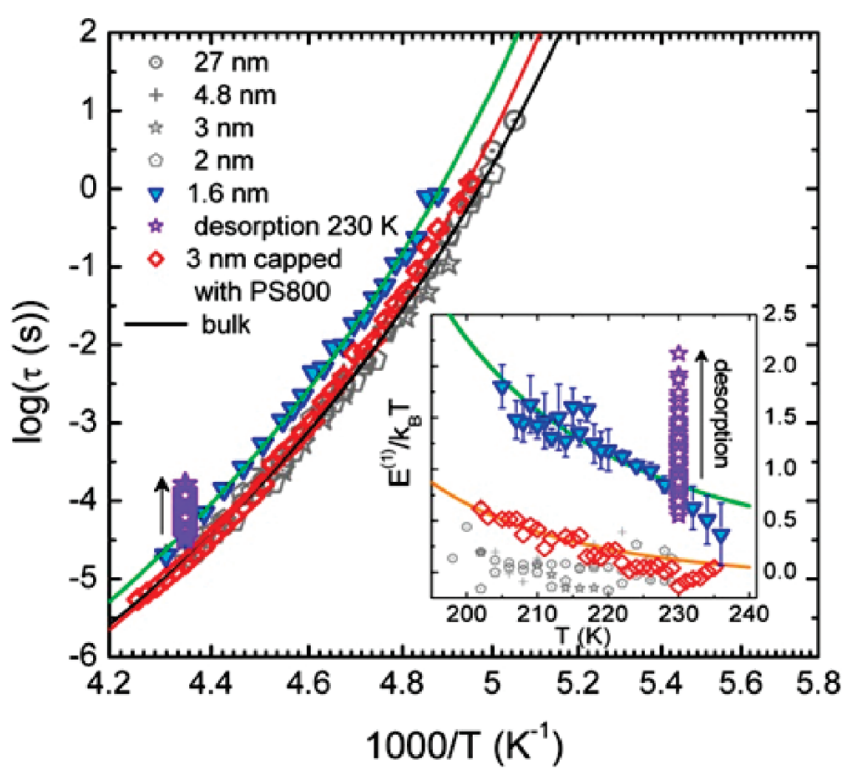

Figure 2. Thermal and thickness evolution of the structural relaxation. Solid lines: VFT fit curves. $B=15$ and $\tau_{\infty}=10^{-15} \mathrm{~s}$ for all films. $T_{0}=144 \mathrm{~K}$ at $1.6 \mathrm{~nm}$, and $T_{0}=139 \mathrm{~K}$ for all of the other thicknesses. $T_{0}=142 \mathrm{~K}$ for the $3 \mathrm{~nm}$ capped film. Inset: perturbation of the activation energy $E^{(1)}$ in units of $k_{\mathrm{B}} T$.

deposited layer is monitored by a quartz crystal microbalance (QCM), located close to the IDE sensor inside the chamber. The dielectric response of the film is continuously acquired at frequencies between $1 \mathrm{~Hz}$ and $1 \mathrm{MHz}$ by means of a highresolution dielectric analyzer (ALPHA N, Novocontrol Technologies). The temperature of the sample is controlled by combined action of a resistance heating and a cooling flux of gaseous nitrogen.

Differently from the parallel plate configuration, commonly used for the investigation of ultrathin polymer films, the direction of the probing electric field in the IDE is parallel to the surface of the film (see Figure 1c). As a consequence, the effective dielectric response is the linear convolution of the contributions of the molecular layers constituting the film ${ }^{16}$ (see Figure 1c). The dielectric loss spectra $\varepsilon^{\prime \prime}(\omega)$ were analyzed in the frequency domain using the empirical Havriliak-Negami (HN) function

$$
\varepsilon^{\prime \prime}(\omega)=\operatorname{Im}\left\{\frac{\Delta \varepsilon}{\left[1+(1+i \omega \tau)^{a}\right]^{b}}\right\}
$$

where $a$ and $b$ express respectively the width and the asymmetry of the relaxation peak, $\Delta \epsilon$ is the dielectric strength, $\omega$ is the angular frequency, and $\tau_{\mathrm{HN}}$ is the relaxation time.

The temperature dependence of $\tau$ for films in the thickness range between 27 and $1.6 \mathrm{~nm}$, measured after deposition, is given in Figure 2. The values of $\tau$ have been estimated according to the fit independent relation $2 \pi f_{\max } \tau=1$, where $f_{\max }(=\omega / 2 \hbar)$ is the frequency of the peak maximum in the dielectric loss. Subsequently, we have fitted the values $\tau(T)$ for all measured films to the Vogel Fulcher Tamman (VFT) equation

$$
\tau=\tau_{\infty} \exp \left[B T_{0} /\left(T-T_{0}\right)\right]
$$

where $T_{0}$ is the Vogel temperature and $B$ is a parameter related to the dynamic fragility of the system.

Films of thickness between 2 and $27 \mathrm{~nm}$ (gray symbols) showed an almost identical temperature dependence $\tau(T)$ compared to bulk glycerol (black line). The value of the glass transition temperature $T_{\mathrm{g}}$, considered as the temperature at which $\tau=100 \mathrm{~s}$, is consequently constant within the experimental error. The value determined via this approach $(193.8 \mathrm{~K})$ is in agreement with previous measurements in bulk. ${ }^{17-19}$ However, the thinnest film with a mean thickness of $1.6 \mathrm{~nm}$ revealed a general slowdown in the relaxation times with respect to the other films, which expresses in a higher Vogel temperature $T_{0}$ (shifted by $+5 \mathrm{~K}$ ) and a corresponding increase of $T_{\mathrm{g}}$ by $3.5 \mathrm{~K}$.

On the contrary, the parameters $B$ and $\tau_{\infty}$ remained unchanged. The same trend was observed during isothermal desorption at $230 \mathrm{~K}$ (from 3 to $0.7 \mathrm{~nm}$ at a rate $\sim 0.4 \mathrm{~nm} / \mathrm{h}$ ) where the structural relaxation time increased by almost 1 order of magnitude. Similar results were obtained upon confinement in Vycor glasses with pore sizes of $7 \mathrm{~nm} .{ }^{20}$ The picture obtained from temperature dependent experiment is the signature of the presence of a reduced mobility layer (RML) in close proximity of the substrate, characterized by slower dynamics. ${ }^{5}$ The existence of a RML in our systems can be ascribed to the attractive $\mathrm{H}$-bonding interactions between the adsorbed glycerol molecules and the substrate of the IDE sensor.

Small variations of the Vogel temperature accompanied by constant values of $B$ and $\tau_{\infty}$, indicate the presence of a molecular population relaxing as in bulk (same dynamic fragility) but with a slightly modified activation energy. In particular, in the framework of an energy landscape scenario, the specific case of increase of $T_{0}$ can be rationalized as a vertical shift of the potential wells reducing the probability for rotational jumps, without affecting the contour of the landscape itself. The resulting increase in the relaxation times can be used to quantify the effects of interfacial interactions, via a perturbation approach of the temperature dependence of the activation energy ${ }^{5}$

$$
E^{(1)}(h, T) \approx[E(h, T)-E(\infty, T)]=k_{\mathrm{B}} T \ln \left[\frac{\tau_{\alpha}(h, T)}{\tau_{\alpha}(\infty, T)}\right]
$$

where $h$ is the thickness of the film and $\infty$ indicates bulk values. Values of $E^{(1)}$ for all the films are reported in the inset of Figure 2 where we used the same symbols as in the main panel. $E^{(1)}$ is clearly nonzero during the latest stages of the desorption at 230 $\mathrm{K}$ and for the whole temperature range at $1.6 \mathrm{~nm}$, where as expected $^{5}$ it increases upon cooling. These results and the concurrent absence of any transition to single molecule dynamics (Arrhenius behavior) as observed for glycerol confined to silica matrices with $2.2 \mathrm{~nm}$ pores, ${ }^{21}$ provide strong evidence for interfacial interactions being the exclusive origin for "confinement effects" in our case. Differently from films probed by an electric field parallel to the direction of confinement (conventional dielectric spectroscopy), we did not observe any reduction of the dielectric strength in the thinnest films. The reduction of dielectric strength due to a restriction in the molecular mobility is probably compensated by a more efficient coupling between the neat dipole moment of the molecule (pointing in the plane perpendicular to the alkyl chainbone) and the electric field. The absence of a strong reduction of dielectric strength suggests an enrichment of chain population where the alkyl chains lie perpendicular to the substrate. These molecular conformations are probably the effect of the steric and electronic hindrance induced by adsorption of outer $\mathrm{OH}$ groups inhibiting multiple bonding within the same molecule.

In addition to a slight increase of the overall activation energy, the (local) perturbations of the molecular mobility in proximity 


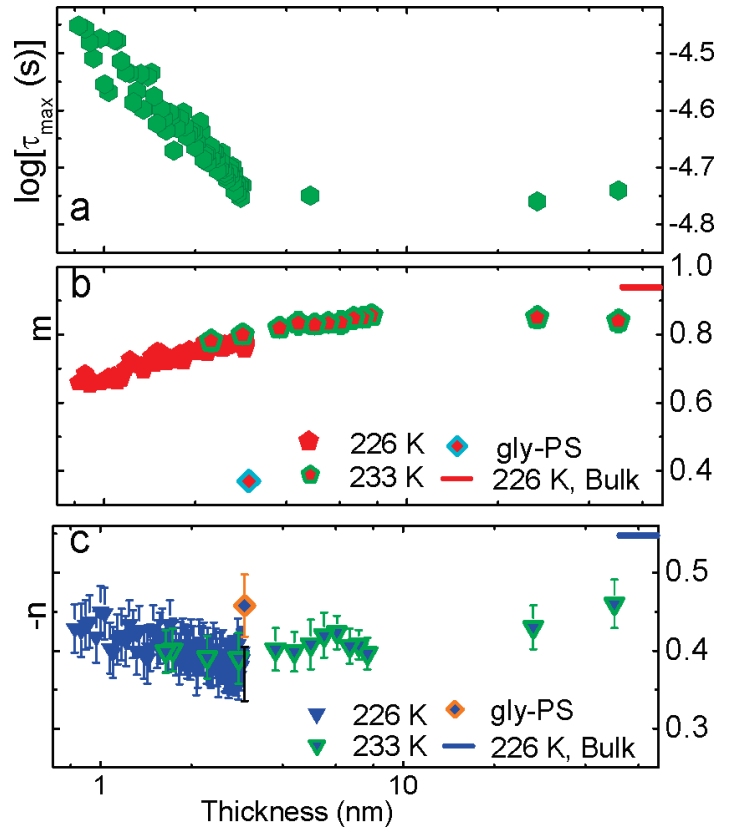

Figure 3. Panel a: thickness dependence of the structural relaxation time, measured during isothermal desortpion at $226 \mathrm{~K}$. Panel b: parameters $m$ and $n$ obtained from two distinct measurements during isothermal desorption: at $233 \mathrm{~K}$, in the thickness range between 2.5 and $8 \mathrm{~nm}$, and at $226 \mathrm{~K}$, between 3 and $0.7 \mathrm{~nm}$.

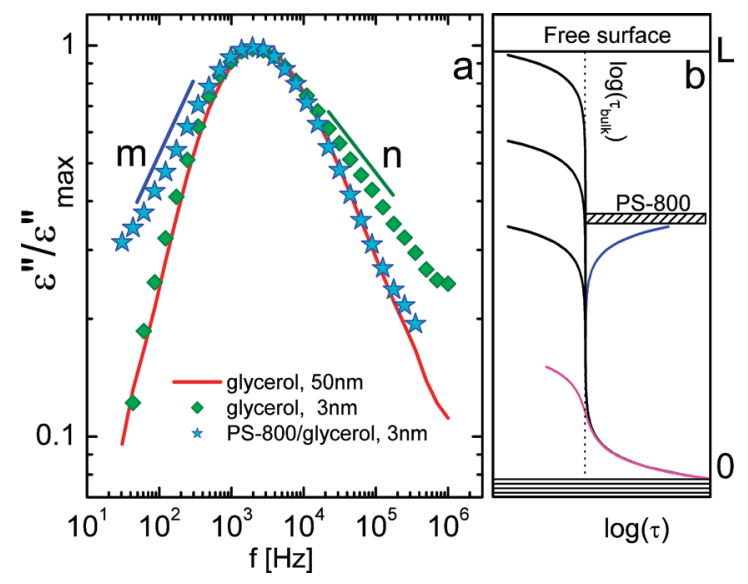

Figure 4. Left panel: Dielectric loss curves acquired for a $3 \mathrm{~nm}$ glycerol film at $217 \mathrm{~K}$, before and after capping with PS800. compared with a $50 \mathrm{~nm}$ thick film (blue line). Right panel: Sketch of mobility profiles across the film, for different values of the total thickness. The blue curve represents the profile in a capped film.

of interfaces induces an increase in the dynamic heterogeneity of the system, which results in a broadening of the distribution of the relaxation times. As the RML and the free surface produce opposite effects on the mean molecular dynamics, we can distinguish their particular contributions by means of the "shape" parameters $m=a$ and $-n=a \cdot b$, which provide the limiting slope of $\log \left(\varepsilon^{\prime \prime}\right)$ vs $\log (\omega)$ at low and high frequencies respectively; see Figures 3 and 4 . The value of $m$ is also related to the width of the distribution of the relaxation times, which is sharper for higher values of $m$ and is limited to one single relaxation time (Debye model) for $m=1$. Values of $m$ and $n$ for bulk glycerol indicate a quite sharp and asymmetric peak, and show a very weak temperature dependence. ${ }^{17,22}$ Reductions in $m$ or in $n$ reflect respectively an enrichment of the distribution of relaxation times at long time scales (slower dynamics) induced by the presence of an RML or short time scale (faster dynamics), by a free surface.
Films were schemed as a trilayer, composed by an RML, an enhanced mobility layer (EML) in vicinity of the free surface, and a bulk like region embedded in between. The impact of the nonbulk component is expected to increase upon thickness reduction, resulting in a reduction of $m$ and $n$. Our experimental results highlight an asymmetric broadening of structural peak toward longer relaxation times.

Figure 3 shows the thickness evolution of the values of $m$ and $n$ obtained from spectra acquired during two distinct continuous film thinning experiments, ${ }^{23}$ i.e., during isothermal desorption at 233 and $226 \mathrm{~K}$; at this last temperature, a lower desorption rate allows higher thickness resolution. Good reproducibility was observed in the overlapping range covered by both experiments.

All three panels in the Figure 3 indicate the presence of two regimes. Below $3 \mathrm{~nm}$ the relaxation times get longer, while the value of $m$ decreases already at $6-8 \mathrm{~nm}$. Thus the increase in $\tau$ is caused by a more pronounced dielectric response at low frequencies, i.e., a larger impact of the RML on the relaxation dynamics of the system upon thickness reduction. The absolute value of $n$, on the contrary, decreases already at few tens of $\mathrm{nm}$, shows a broad minimum centered around $3 \mathrm{~nm}$, and recovers "bulk" values below $1 \mathrm{~nm}$. The different thickness dependence of the two shape parameters implies an asymmetric peak broadening upon film thinning, i.e., changes of $m$ and $n$ are caused by a variation in distribution of the relaxation times rather than to an intrinsic increase in the heterogeneity caused by confinement (which would induce a symmetric broadening).

The analysis of the shape parameters $m$ and $n$ suggest that upon film thickness reduction the free surface gradually approaches the RML and its mobility gets enslaved to the slow dynamics. This evidence is consistent with the idea that the glass transition temperature of a free surface is bound to the nature of the underlying layer. ${ }^{24}$ In films thinner than $3 \mathrm{~nm}$ the more stable boundary conditions imposed by the interactions $(-\mathrm{OH}$ bonding) with a rigid substrate inhibit the acceleration provided by the molecules in contact with vacuum at the upper interface that alters the high frequency response of glycerol for layer thicknesses smaller than $50 \mathrm{~nm}$. In Figure 4b, we sketched possible mobility profiles capturing the concurrent effects of a RML and an enhanced mobility layer on the overall dynamics.

To further focus on the contribution of regions with faster dynamics in glycerol and their impact on the structural relaxation we measured the response of a capped film (Figure 4a), where the free surface is suppressed. We deposited a layer $(9 \mathrm{~nm})$ of low molecular weight polystyrene $\left(M_{\mathrm{w}}=800 \mathrm{~g} / \mathrm{mol}, T_{\mathrm{g}}=269\right.$ K), PS-800, on top of a $3 \mathrm{~nm}$ thick film of glycerol. Spectra of the capped film were acquired upon heating and compared to measurements performed on the underlying glycerol layer before capping. As PS-800 does not present any relaxation process in the chosen temperature range, any difference in the relaxation pattern of the two measurements can be attributed to a different nature of the interfaces. Measurements were performed below the $T_{\mathrm{g}}$ of PS-800, where the extremely low miscibility with glycerol ensures geometrical stability of the system for the whole duration of the experiment. Loss curves for both the capped and the uncapped film, acquired at $217 \mathrm{~K}$, are displayed in Figure 4a. An additional RML is introduced at the upper interface, as proved by the further reduction of $m$. The change in the intermolecular interactions involving the molecules at the very interface with PS-800 resulted in longer relaxation times (Figure 2, red open diamonds) and a nonzero confinement energy (Figure 2, inset), at a thickness on the order of the double of the threshold measured for films with a free surface. The 
comparison between the dielectric spectra before and after capping revealed a depletion of faster molecules in the capped film. In Figure 4 the loss curve measured at the same temperature for a $50 \mathrm{~nm}$ thick film is also reported for comparison. The relaxation peak of the $3 \mathrm{~nm}$ capped film overlaps the high frequency component of the peak of the thicker film, and consequently the value of $-n$ of the two samples are equal. This evidence proves that the free surface is suppressed by depositing a capping layer.

The suppression of the free surface results in a reduction of the relaxation peak component at high frequencies by a component whose dielectric strength corresponds roughly to one forth of the overall signal of the uncapped glycerol film; that is, the effects of the free surface on glycerol are limited to a monolayer. The small extension of the enhanced mobility layer and the increasing values of $-n$ upon reduction of the thickness (Figure $3 b$ ) prove that the impact of the of the free surface on the overall relaxation time of thin films is lower than that one of the RML, and rationalizes the slowdown of dynamics observed at $1.6 \mathrm{~nm}$.

Acknowledgment. S.C. acknowledges financial support from the Research Council of the K.U. Leuven, Project No. OT/ 30/06. S.N. acknowledges FWO (Fonds Wetenschappelijk Onderzoeks - Vlaanderen) for a postdoctoral scholarship. M.W. acknowledges financial support from FWO within the Project G.0642.08.

\section{References and Notes}

(1) Arndt, M.; Stannarius, R.; Gorbatschow, W.; Kremer, F. Phys. Rev. E 1996, 54, 5377.

(2) Arndt, M.; Stannarius, R.; Groothues, H.; Hempel, E.; Kremer, F. Phys. Rev. Lett. 1997, 79, 2077.
(3) Huwe, A.; Kremer, F.; Behrens, P.; Schwieger, W. Phys. Rev. Lett. 1999, 82, 2338.

(4) Ngai, K. L. J. Non-Cryst. Solids 2000, 275, 7.

(5) Napolitano, S.; Lupascu, V.; Wubbenhorst, M. Macromolecules 2008, 41, 1061.

(6) Napolitano, S.; Pilleri, A.; Rolla, P.; Wubbenhorst, M. ACS Nano 2010, 4, 841 .

(7) Napolitano, S.; Prevosto, D.; Lucchesi, M.; Pingue, P.; D'Acunto, M.; Rolla, P. Langmuir 2007, 23, 2103.

(8) Alcoutlabi, M.; McKenna, G. B. J. Phys.-Condesed Matter 2005, 17, R461.

(9) Li, Q. X.; Simon, S. L. Macromolecules 2009, 42, 3573.

(10) Koh, Y. P.; Li, Q.; Simon, S. L. Thermochim. Acta 2009, 492, 45.

(11) Laureyn, W.; Nelis, D.; Van Gerwen, P.; Baert, K.; Hermans, L.; Magnee, R.; Pireaux, J. J.; Maes, G. Sens. Actuators B-Chem. 2000, 68, 360.

(12) Scott, M. C.; Stevens, D. R.; Bochinski, J. R.; Clarke, L. I. ACS Nano 2008, 2, 2392.

(13) Rotella, C.; Napolitano, S.; Wuebbenhorst, M. Macromolecules 2009, 42, 1415.

(14) Measurements on films below $1 \mathrm{~nm}$ could not be performed over a wide temperature range due to film instability.

(15) Sharp, J. S.; Forrest, J. A. Phys. Rev. Lett. 2003, 91, 235701/1.

(16) Peter, S.; Napolitano, S.; Meyer, H.; Wubbenhorst, M.; Baschnagel, J. Macromolecules 2008, 41, 7729.

(17) Lunkenheimer, P.; Pimenov, A.; Schiener, B.; Bohmer, R.; Loidl, A. Europhys. Lett. 1996, 33, 611.

(18) Mobius, M. E.; Xia, T.; van Saarloos, W.; Orrit, M.; van Hecke, M. J. Phys. Chem. B $, 114,7439$.

(19) Zondervan, R.; Kulzer, F.; Berkhout, G. C. G.; Orrit, M. Proc. Natl. Acad. Sci. U. S. A. 2007, 104, 12628.

(20) Kilburn, D.; Sokol, P. E.; Sakai, V. G.; Alam, M. A. Appl. Phys. Lett. 2008, 92, 1.

(21) Levchenko, A. A.; Jain, P.; Trofymluk, O.; Yu, P.; Navrotsky, A.; Sen, S. J. Phys. Chem. B 2010, 114, 3070.

(22) Schneider, U.; Lunkenheimer, P.; Brand, R.; Loidl, A. J. Non-Cryst. Solids 1998, 235, 173.

(23) Both the values of $m$ and $n$ are intrinsically lower than those obtained for bulk glycerol probed with an isotropi electric field, for thicknesses at least up to $50 \mathrm{~nm}$; this discrepancy can be ascribed to the different geometrical configuration adopted for the thin films (see ref 16).

(24) Roth, C. B.; Torkelson, J. M. Macromolecules 2007, 40, 3328.

JP108151P 